\title{
Mejoramiento sostenible de la calidad de vida de la población mediante el trabajo comunitario
}

\section{Sustainable improvement of the quality of life of the population based on the community-wide work}

\author{
Rhoden N. Rodríguez Casavielles,' Oscar L. Bellido Aguilera," Claribel \\ González Calzadilla," 'I Ediltrudis Solares Sierra," I I rma Rojas Verdecia," \\ Rafael Lorenzo Martínvı \\ ' Doctor en Medicina. Especialista de I Grado en Higiene y Epidemiología. Centro \\ Provincial de Higiene, Epidemiología y Microbiología. Holguín, Cuba. \\ "Arquitecto. Universidad de Holguín. Holguín, Cuba. \\ III Licenciada en Educación. Máster en Gerencia de la Ciencia y la Innovación. \\ Delegación Provincial del CITMA. Holguín, Cuba. \\ IV Licenciada en Filosofía. Máster en Ciencias. Universidad de Holguín. Holguín, Cuba. \\ $\checkmark$ Licenciada en Educación. Universidad de Holguín. Holguín, Cuba. \\ VI Licenciado en Educación. Doctor en Ciencias Pedagógicas. Dirección Municipal de \\ Educación. Holguín, Cuba.
}

\section{RESUMEN}

I ntroducción La iniciativa cubana por la calidad de vida no solo se concreta en indicadores médicos, sino que está asegurada por políticas sociales y mecanismos gubernamentales en una estructuración desde el nivel provincial, municipal hasta los Consejos Populares y sus circunscripciones.

Objetivo Demostrar la importancia del trabajo comunitario como vía segura para mejorar la calidad de vida de las personas.

Métodos Se decidió actuar en el Consejo Popular "Alcides Pino Bermúdez", en el municipio Holguín, por sus características. Se realizó revisión documental y una investigación-acción participativa, desde el 2009, donde se identificaron los problemas personales, materiales y de mediación, estos últimos, consecuencia directa de insuficiencias en la integración intersectorial.

Resultados Se constató la necesidad de la presencia de un Grupo de Trabajo Comunitario capacitado. Se comprobó que el mantenimiento sostenible de la calidad 
de vida de la comunidad no puede ser un asunto espontáneo de personas, organismos u organizaciones.

Conclusiones Es preciso contar con Grupos de Trabajos Comunitarios capacitados en sus funciones. El actuar por la calidad de vida de la comunidad debe sustentarse en elementos científicos, con fines concretos y conducidas por personas capaces.

Palabras clave: Calidad de vida, trabajo comunitario, intersectorialidad.

\begin{abstract}
Introduction The Cuban initiative for the quality of life is not only materialized in medical indicators but also supported by the social policies and the governmental mechanisms within a structuring going from the province, the municipality to the people's councils and their constituencies.

Objective To prove the importance of the community-wide work to improve the quality of life of the people.

Methods It was decided to work in "Alcides Pino Bermúdez" people's council in Holguín municipality because of its characteristics. Documentary review and participatory action research were made since 2009, which resulted in detection of personal, material and mediatory problems, being the last direct consequence of inter-sectoral integration shortcomings.

Results It was confirmed that a trained community work group was necessary. Working for the quality of life of the community can no longer be a spontaneous task undertaken by individuals, bodies or organizations; it should be based on scientific elements, performed by trained people and directed to specific targets.
\end{abstract}

Conclusions It is required to have community work groups trained in performing their functions, particularly in the sustainability of the quality of life of the community.

Key words: Quality of life, community-wide work, inter-sectorality.

\title{
I NTRODUCCI ÓN
}

En las consideraciones iniciales acerca del Proyecto de Lineamientos de la Política Económica y Social, del VI Congreso del Partido Comunista de Cuba, se identifica la necesidad de impulsar con efectividad la iniciativa de los territorios (por tanto, de las comunidades) para potenciar de manera sostenible su desarrollo económico (cabe añadir, su desarrollo comunitario), se reitera que el trabajo debe ser motivo de realización personal para cada ciudadano, y se precisa que las soluciones del desarrollo sostenible deben permitir un uso eficiente del potencial humano en las comunidades cubanas. ${ }^{1}$

Más adelante, en los lineamientos generales de la política social se explicita la intención de: continuar preservando las conquistas de la Revolución en las principales esferas de servicios tributarias directas del mejoramiento de la calidad de vida, rescatar el papel del trabajo como la vía fundamental para contribuir al desarrollo de la sociedad (por consiguiente, de la comunidad) y a la satisfacción de las necesidades personales y familiares, así como garantizar la elevación sistemática y sostenida de la 
calidad de los servicios que se brindan a la población y dar continuidad al perfeccionamiento de la educación, la salud, la cultura y el deporte, componentes fundamentales de la calidad de vida del pueblo cubano. ${ }^{1}$

Desarrollo, desarrollo sostenible, realización personal, satisfacción de necesidades humanas, calidad de vida, trabajo creador, potencialidades humanas, iniciativa ciudadana y organizacional, son conceptos interactúantes e interdependientes en un entrelazamiento dialéctico sistémico complejo con manifestaciones concretas en la vida cotidiana de cualquier comunidad del país.

¿Cómo lograr que los términos "desarrollo comunitario sostenible", "calidad de vida" y otros cercanos a estos, sean algo más que conceptualizaciones solo del dominio de especialistas, y se asuman en las comunidades en tanto dimensiones esenciales de su vida cotidiana pero, sobre todo, como asuntos de su responsabilidad (compartida) que exigen para su concreción del compromiso y la participación de cada persona como sujeto individual y de la comunidad toda en su condición de sujeto colectivo de su propio desarrollo?

La interrogante anterior es una de las formuladas en el proceso de investigaciónacción en marcha en la provincia de Holguín como parte del desarrollo constante de la iniciativa cubana por la calidad de vida a través de la política social y mecanismos gubernamentales en una estructuración desde el nivel provincial, municipal hasta los consejos populares y sus circunscripciones, sustentado en las potencialidades particulares existentes en cada entorno para lograr una integración de todos los factores, sobre la base de la responsabilidad individual, la multidisciplinaridad, fomentando el papel clave de los delegados de circunscripción con su grupo de trabajo comunitario como agentes que centren la conducción de los procesos.

\section{Procesos del desarrollo}

El desarrollo como proceso universal guarda una dialéctica que encierra el desarrollo social, comunitario y humano, y que impone ser estudiado desde una determinada posición filosófica, en este caso, la del materialismo dialéctico pues ofrece la explicación más coherente, fundamentada y no lograda por otros hasta el momento, y sus premisas permiten crear una teoría social capaz de interpretar, fundamentar y proponer nuevas alternativas de solución para los problemas de la realidad actual. ${ }^{2}$

El desarrollo social, entendido como el proceso de múltiples acciones conscientes de los hombres en determinados contextos socioculturales con el fin de transformarlos para garantizar mejores condiciones de vida, más bienestar y una calidad de vida superior en correspondencia con sus necesidades básicas reales. ${ }^{2}$

Así, cuando el "contexto sociocultural" es la comunidad se trata de desarrollo comunitario, interpretado en el sentido de un proceso por medio del cual la población de un asentamiento concebido como comunidad se integra, a partir de sus potencialidades, en la transformación de su entorno, teniendo como bases determinantes la participación, el liderazgo, la planificación, la organización, la capacitación y la dialéctica entre centralización y descentralización, factores indispensables para mejorar las condiciones económicas, sociales y culturales de cada comunidad, favorecer el desarrollo humano local e incorporarlas al desarrollo social a escala nacional. ${ }^{2}$ Nace de una política y una estrategia previstas a nivel macro por el Estado, aspecto que marca de forma significativa los alcances de este proceso.

El desarrollo comunitario así concebido exige ser conducido hacia sus fines mediante un proceso de gestión o trabajo comunitario integrado (planeamiento comunitario, organización, ejecución, control/evaluación), sistémico, es decir, un proceso de 


\section{Revista Cubana de Salud Pública. 2011;37(3):219-229}

autoconducción donde se integran sistémicamente los diferentes niveles de dirección social del país: nacional, provincial, municipal, del consejo popular y de la propia comunidad.

Por su parte, el concepto de desarrollo humano supone un cambio radical respecto de lo que ha sido la preocupación central de la economía del desarrollo; cuestiona la premisa de que el desarrollo es el mero aumento de la producción de bienes y servicios; propone el desarrollo como un proceso de ampliación de las capacidades de las personas; cuestiona el presupuesto de que el medio para alcanzar el desarrollo es solamente la acumulación de capital físico, y plantea la necesidad de invertir recursos para alcanzar otros objetivos humanos. ${ }^{3}$ De esta forma, el concepto de desarrollo humano se entiende como la expansión de las libertades reales disfrutadas por las personas para elegir las cosas consideradas valiosas por ellas, o para eliminar fuentes de privación como la pobreza económica, la falta de servicios, la violación de la libertad. Denota tanto el proceso de ampliar las oportunidades de los individuos, como el nivel de bienestar que estos han alcanzado.

En relación con lo anterior, es posible distinguir dos aspectos: uno, la formación de capacidades humanas tales como un mejor estado de salud o mayores conocimientos $y$, dos, la forma como los individuos emplean las capacidades adquiridas ya sea para el trabajo o para el descanso. ${ }^{3}$

El desarrollo humano entraña mucho más que el simple aumento o disminución del ingreso nacional. Significa crear un entorno donde las personas puedan hacer plenamente realidad sus posibilidades y vivir en forma productiva y creadora de acuerdo con sus necesidades, aspiraciones e intereses. Los pueblos son la verdadera riqueza de las naciones $y$, por ende, el desarrollo debe favorecer la ampliación de sus opciones para vivir de acuerdo con sus valores. Por eso el desarrollo debe ir más allá del crecimiento económico, el cual solo constituye un medio, aunque muy importante, para ampliar las opciones de la población. ${ }^{4}$

Un desarrollo "a escala humana" tendría tres pilares, a saber: la satisfacción de las necesidades humanas fundamentales, la generación de niveles crecientes de autodependencia o autoconducción y la articulación dialéctica y sistémica de los seres humanos con la naturaleza y la tecnología; los procesos globales con los comportamientos locales y comunitarios, lo personal con lo comunitario y lo social, la planificación con la autonomía o la centralización con la descentralización, la sociedad civil con el Estado.

La base de tales pilares debiera ser el protagonismo real de las personas, cuya construcción exige las siguientes condiciones: la diversificación y autonomía de espacios facilitadores de dicho protagonismo, la ausencia de sistemas sólo organizados jerárquicamente desde arriba hacia abajo, y el cambio del rol paternalista del Estado por otro estimulador de soluciones creativas emergentes desde la comunidad hacia arriba y, por tanto, más congruentes con las aspiraciones reales de las personas. ${ }^{5}$

Max-Neef M, se pregunta cómo saber cuándo un proceso de desarrollo es mejor que otro, y responden que el mejor proceso de desarrollo es aquel donde se eleve más la calidad de vida de las personas; esta dependerá, a su vez, de las posibilidades al alcance de las personas para satisfacer adecuadamente sus necesidades humanas fundamentales. Ahora bien, ¿cuáles son esas necesidades humanas fundamentales y quiénes las determinan ${ }^{5}$ 
El autor citado postula que las necesidades humanas fundamentales son finitas, pocas, clasificables y las mismas en todas las culturas y períodos históricos, pues lo que varía, casi hasta el infinito, son los llamados satisfactores* de esas necesidades.

\section{Calidad de vida, necesidades humanas y problema comunitario}

El indicador cualitativo "calidad de vida" incluye el estado de salud física y mental, el estilo y los modos de vida, la satisfacción con la existencia individual y familiar y el grado de bienestar autovalorado de su situación en la vida, dentro del contexto cultural y valores donde se desenvuelve, en relación con sus objetivos, aspiraciones, expectativas e intereses.

Se trata de las condiciones de vida de una comunidad o individuo que al evaluarlas desde los puntos de vista de una racionalidad reproductiva (que asegura el ciclo natural de la vida), una ética de la responsabilidad (individual, familiar, comunitaria, social) y de acuerdo con criterios técnicos, legales, morales, axiológicos, estéticos, de la cultura y la persona; se llega al consenso de ser coherentes con el progreso, la adaptación, la sobrevivencia y el bienestar colectivo e individual.

Max-Neef clasifica y combina las necesidades en dos categorías: las existenciales son las necesidades de: ser, tener, hacer y estar; y las axiológicas; las de: subsistencia, protección, afecto, entendimiento, participación, ocio, creación, identidad y libertad. Así, se pueden definir como la manera más apremiante en que se revela el ser de las personas, el cual se hace palpable a través de la doble condición existencial de estas: como carencia y como potencialidad. Comprendidas en un amplio sentido, y no limitadas a la mera subsistencia, las necesidades patentizan la tensión constante entre carencia y potencia tan propia de los seres humanos. ${ }^{5}$

Asimismo, como la persona es un ser de necesidades múltiples e interdependientes, estas deben asumirse como un sistema donde se interrelacionan e interactúan. Por consiguiente, como estructura sistémica y proceso dialéctico, constituyen una unidad inseparable en movimiento incesante, de ahí que el tratamiento de las necesidades comunitarias demande un abordaje transdisciplinar, integral y no fragmentado.

Cada sistema social genera vías para la satisfacción de las necesidades humanas fundamentales. Estas se alcanzan (o no) a través de la generación (o no) de diferentes tipos de satisfactores.* Desde lo cultural no son las necesidades humanas fundamentales, sino sus satisfactores los que se construyen.

En resumen, cabe entender las necesidades humanas como contradicciones cuyos polos positivo y negativo (en el sentido de que lleva en sí la negación de lo existente) son la carencia y la potencialidad, respectivamente, mientras los satisfactores son los factores que median entre tales polos y forman parte inseparable de la contradicción o unidad dialéctico-sistémica como "lo tercero" que la completa.

Con la anterior explicación teórica se persigue resaltar un aspecto considerado esencial para la práctica del trabajo comunitario: el imperativo de tratar tanto las carencias como las potencialidades y los satisfactores, y hacerlo de forma integrada, en la identificación de los problemas de la comunidad y la búsqueda de soluciones para ellos.

Comprender a las personas en función de las necesidades consideradas como carencia y potencia, previene contra toda reducción del ser humano a la categoría de existencia cerrada. 
Un concepto operacional para el trabajo comunitario, es el de "problema comunitario"**, definido como una "carencia" insatisfecha, una "aspiración" irrealizada o una "potencialidad" desaprovechada, por tanto, un obstáculo para el avance hacia los fines de la comunidad que puede producir involución, conflicto y frustración pero, a la vez, capaz de potenciar el "compromiso", la "motivación", la "movilización", la creatividad y la innovación en las personas, y contribuir al desarrollo humano y al desarrollo comunitario si se identifica con precisión y se actúa con imaginación, voluntad y eficiencia para resolverlo.

Los aspectos anteriores fueron la motivación esencial para realizar esta investigación en la provincia Holguín, en el consejo popular "Alcides Pino Bermúdez", con el objetivo de diseñar e implementar acciones de diagnóstico, conducción de procesos y de solución de sus problemas a partir del trabajo comunitario como vía segura para mejorar la calida de vida de la población. Se decidió actuar en este consejo por las características que presenta (anexo1).

En el anexo 2 aparecen las premisas a considerar en el desarrollo de políticas concretas en el contexto actual, para mejorar la calidad de vida de los ciudadanos de forma sostenible.

\section{MÉTODOS}

Con los presupuestos conceptuales expuestos hasta aquí, en el mes de septiembre de 2009 se inició el trabajo en el consejo "Alcides Pino Bermúdez", con la participación coordinada de todas las instituciones y organizaciones involucradas de una manera u otra en el proceso de cambio, conducido por las autoridades de gobierno y políticas del territorio, previendo un respaldo en recursos materiales y financieros que permitiera emprender un proceso inversionista orientado básicamente hacia los principales problemas de naturaleza material a nivel de consejos populares en los diferentes municipios (acueducto, alcantarillado, vivienda, viales, electricidad, comunicaciones y otras), identificados en los diagnósticos de los correspondientes organismos, del gobierno municipal y del propio consejo popular, elaborados con anterioridad.

Como complemento del proceso constructivo, se creó un equipo de trabajo coordinador multidisciplinario, con la participación de instituciones científicas y dirigido por el CITMA, representativo de los principales organismos involucrados, conformándose al mismo tiempo, grupos de especialistas que trabajarían en estrecha relación con el referido equipo, iniciándose así una investigación-acción acerca de los problemas sociales y las vías para su progresiva solución, bajo el entendido de que en esta esfera las transformaciones requieren de mayor estudio y de plazos de ejecución más dilatados.

Caracterizado el consejo (anexo1) y luego de varias sesiones de trabajo entre los miembros del equipo y delegados del consejo, se realizó una revisión documental para conocer en teoría que se había hecho al respecto en el territorio y se elaboró un proyecto de investigación-acción participativa. Esta investigación se desarrolló en siete foros comunitarios, en 10 de las 15 circunscripciones del consejo, con la participación de 608 personas, incluidos los delegados de circunscripciones y los miembros del equipo coordinador. 


\section{RESULTADOS}

Los miembros de la comunidad participantes en los foros formularon 207 problemas en total y, según su caracterización más de la mitad (59\%) eran "de mediación"***. Asimismo, los problemas materiales formulados representaron el $27 \%$ del total, y los problemas personales el $14 \%$.

Los problemas más reiterados, autoidentificados en la mitad o más de las circunscripciones en cada caso, entre los personales o subjetivos, fueron: alto grado de adicción al alcohol, desvinculación del trabajo, insuficiencias en las escalas de valores individuales (generan malas prácticas comunitarias y de convivencia social); entre los materiales: insuficiencia de infraestructura para la recreación y los deportes (especialmente para niños), déficit de servicio de alcantarillado, insuficiencia de teléfonos públicos, mal estado de los viales, señalización insuficiente y falta de mantenimiento de las señales existentes (lo que implica peligros, sobre todo para los niños en horarios escolares), falta de una agencia bancaria y de una funeraria, insuficiente iluminación pública.

Y, entre los de mediación: insuficientes espacios u opciones para el uso del tiempo libre (al margen de las disponibilidades de instalaciones), inestabilidad en la atención de los médicos del consultorio y poco trabajo de terreno, deficiencias en el servicio de acueducto (ciclo y horario del agua, independiente de la disponibilidad de agua y redes), deficiente gestión de saneamiento y limpieza de fosas por comunales (no relacionado con el déficit y mal estado técnico de los equipos), inestabilidad en la calidad y distribución del pan (no dependiente de los problemas de calidad de las materias primas).

Un aspecto fundamental derivado del proyecto de investigación-acción participativa es la propuesta de implementación de un Plan General del Consejo Popular, instrumento básico de la integración, documento rector en la conducción del proceso de autogestión comunitaria en función de la calidad de vida al incluir todas las dimensiones de la vida comunitaria: social, ambiental, económica, cultural, deportiva, recreativa, urbanística, así como todos los niveles de planificación: proyecto, programa, plan.

El Plan General está compuesto por el Triple Fin del Consejo Popular para su desarrollo comunitario, mostrado en la figura, la Estrategia Comunitaria de desarrollo compuesta por diez objetivos estratégicos que deberán guiar el avance hacia tales fines y el Plan de Acción (2010-2015) para materializar la estrategia formulada.

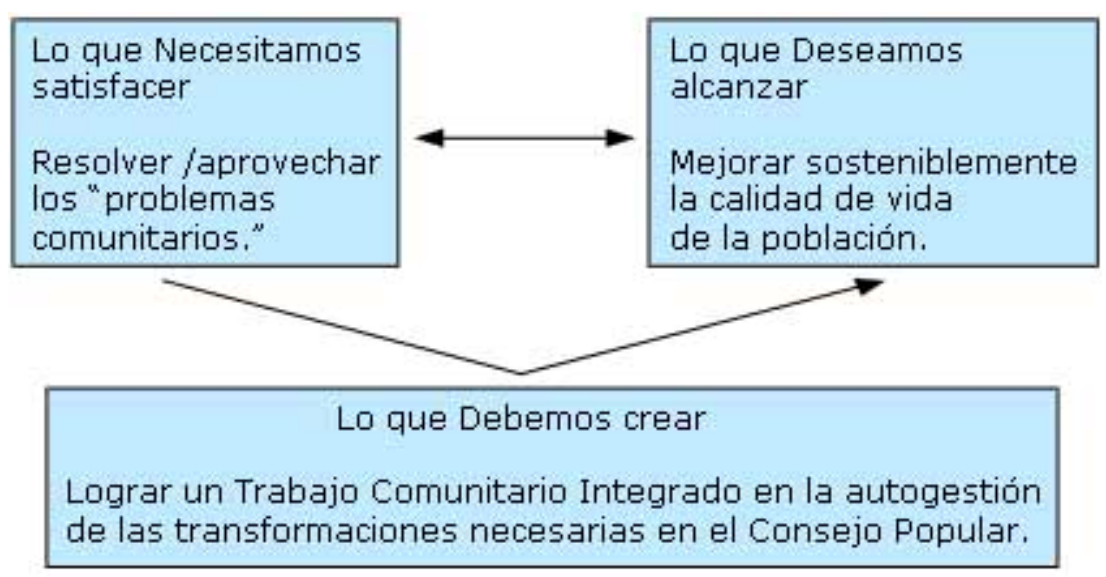

Fig. Triple Fin del Consejo Popular para su desarrollo comunitario. 


\section{DISCUSION}

Según la caracterización de los problemas planteados, en la mayor parte de los casos se trata de "saber" resolverlos, esto es, de falta de comprensión o de capacidades para darles solución, y la mayoría obedecen a insatisfacciones por deficiencias en los procesos de gestión de los referidos sistemas o estructuras.

En la investigación se ratificaron los problemas contenidos en los diagnósticos de los organismos, pero fueron identificados otros problemas no incluidos en estos, lo que permitió lograr una visión más completa de la realidad social del consejo, y demostró la validez de la decisión adoptada en relación con la necesidad que existía de realizar la investigación participativa.

Lo más interesante a destacar en este aspecto es que el procedimiento utilizado posibilitó la identificación por la propia comunidad de los llamados problemas "de mediación"***, no considerados en los referidos diagnósticos, cuestión importante teniendo en cuenta que estos constituyeron más del $50 \%$ del total. Se requiere precisar que las soluciones para este tipo de problemas no están todas al alcance de las posibilidades reales del consejo.

Al respecto, es importante observar que tales problemas de mediación pueden interpretarse como el reflejo o las consecuencias directas de las insuficiencias en la integración, y demuestran la necesidad de implementar mecanismos y procesos de interfase o de gestión de interacciones, como medios auxiliares de las autoridades del consejo, el gobierno municipal y la provincia, medios considerados decisivos en el impulso de los procesos de integración de acciones y de todos los sujetos involucrados, tanto los comunitarios como los municipales y provinciales, para la solución de las necesidades del desarrollo comunitario.

Se demuestra la necesidad de trabajar en escenarios idóneos, con plena participación comunitaria en la identificación de sus problemas en todas las dimensiones, lo que propicia la sensibilización de decisores políticos y de gobierno de la necesidad de tener en cuenta premisas inviolables en el trabajo comunitario como vía segura para mejorar la calidad de vida de las personas.

Se constata que los resultados a obtener y a sostener en la comunidad tienen que ir acompañados de un Grupo de Trabajo Comunitario capacitado pues el actuar por la calidad de vida de la comunidad no puede ser un asunto que se deba a la espontaneidad de personas, organismos u organizaciones, debe sustentarse en elementos científicos, con fines concretos y conducidas por personas capaces. Se decidió la confección del Plan General del Consejo Popular que contiene el Plan de Acción Único como instrumento del Grupo de Trabajo Comunitario en la conducción del proceso integral de la comunidad.

El Plan General se convierte en un instrumento de gobierno, con la aprobación de la Asamblea Municipal del Poder Popular, para la gestión del consejo popular, y en un eslabón no sólo importante sino necesario y de actualidad, del sistema de planificación municipal, teniendo en cuenta la alta prioridad otorgada por la máxima dirección del Estado cubano al "proceso de fortalecimiento de la institucionalidad en el país", en lo que desempeña un importante papel el perfeccionamiento del proceso de planificación pues, "si en épocas normales la planificación es un instrumento imprescindible para la labor de dirección, en el complejo escenario que estamos viviendo se convierte en una necesidad vital que no da espacio a los riesgos de la improvisación y el apresuramiento", precisamente porque excluye la posibilidad de 
dichos riesgos y porque facilita el "enfoque integral que se busca en la actualización del modelo económico cubano". Es preciso caminar hacia el futuro, con paso firme y seguro, porque sencillamente no tenemos derecho a equivocarnos. ${ }^{6}$

Finalmente se puede concluir que es necesario contar con Grupos de Trabajos Comunitarios capacitados en sus funciones, fundamentalmente en cómo lograr el desarrollo sostenible de la calidad de vida de la comunidad, posibilitando esto contar con decisores políticos y administrativos a los diferentes niveles de estructuras existentes, sensibilizados con las premisas indispensables en el trabajo comunitario, con la aplicación de métodos científicos en espacios idóneos para contar con verdaderos instrumentos que permitan la conducción del proceso de autogestión integrada en su comunidad y con ello resultados perdurables en indicadores para mejorar la calidad de vida de las personas.

\section{Anexo 1}

\section{Caracterización del Consejo Popular de "Alcides Pino Bermúdez"}

El Consejo Popular de Alcides Pino está ubicado en el extremo norte de la ciudad de Holguín; limita por el sur con los consejos de Vista Alegre y Centro de Ciudad Norte; por el este, norte y oeste limita con áreas rurales del municipio de Holguín. Se estructura alrededor de un eje vial que lo recorre de sur a norte: la carretera HolguínGibara, y sobre un eje de elevaciones en la dirección oeste-este, formado por la Loma de la Cruz al suroeste y por la Colina de los Internacionalistas al sureste, donde se incluye la Loma de los Guillenes al sur (perteneciente al territorio del Consejo).

En su origen (años 50 del siglo XX y posterior crecimiento, se desarrolló de forma espontánea, sin planificación urbanística alguna, sobre áreas con pendientes poco recomendables para la urbanización, y esto ha generado serias dificultades para la construcción de las infraestructuras técnicas, especialmente del alcantarillado.

Contiene 15 circunscripciones con una población aproximada de 31 mil habitantes $(49,2 \%$ mujeres y 50,8 \% hombres), en una extensión territorial de 10,36 km² para una densidad de más de 2990 personas por kilómetro cuadrado.

Previo al inicio del accionar en el Consejo, el fondo habitacional tenía 8817 viviendas, con $69 \%$ de ellas en mal o regular estado (entre las que se cuentan 2483 con piso de tierra), y un elevadísimo déficit en las redes de alcantarillado y acueducto, así como serias dificultades en las cubiertas, todo ello agravado por el crecimiento descontrolado y en condiciones de precariedad, de los asentamientos de El Paraíso, La Llavita, El Cuncuní, Calle 33 Final y La Garita. La situación en este sector fue priorizada y ha mejorado sustancialmente gracias al Plan en ejecución.

Los diagnósticos sectoriales llaman la atención sobre los siguientes aspectos negativos de la calidad de vida de la comunidad: alcoholismo, hábito de fumar, inadecuada cultura alimentaria, hábitos de vida sedentaria, recreación escasa, desempleo y bajos ingresos salariales, así como algunas manifestaciones de conducta sexual inadecuada, de drogodependencia, de parasuicidio, de conducta social de riesgo, y de violencia familiar y comunitaria.

De igual forma, estos diagnósticos destacan como potencialidades de esta comunidad, el adecuado ambiente sociopolítico, la proyección comunitaria de los centros de salud y educacionales, las instituciones deportivas y las culturales, universalización de la educación superior, entre otras. 


\section{Anexo 2}

\section{Premisas del trabajo por la calidad de vida}

Con los indicadores sociales del territorio, la revisión del papel de los organismos, instituciones y organizaciones, así como debate del tema en foros comunitarios y de expertos, se determinaron las premisas del trabajo por la calidad de vida.

1. Despertar la motivación y el sentido de pertenencia en el actuar de los diferentes actores sociales.

2. Mantener unidad de criterio y de acción en la respuesta social a la solución de los problemas, el accionar a los diferentes niveles debe ser incluyente y participativo, desde su concepción y accionar hasta su evaluación y control.

3. Educar a la comunidad en la autogestión como vía principal en la solución de sus problemas, a partir de la pro actividad y creatividad.

4. Sostenibilidad en el trabajo (preparar a la comunidad al respecto) tener en cuenta que los modos y estilos de vida no se transforman en un día ni en un año para otro, generalmente se produce por cambios generacionales.

5. Ser flexible fundamentalmente desde el punto de vista gerencial y administrativo en el trabajo comunitario, este no tiene límites en tiempo ni espacios.

6. El control y evaluación del trabajo comunitario es imprescindible, proponiendo realizarse en dos direcciones: autoevaluación por el organismo u organización en cuestión (como marcha su accionar, así como su interrelación con el resto de los organismos que intervengan) y la evaluación externa, que debe realizarse por un grupo de trabajo comunitario preparado para ello o por propuestas que haga este grupo o por expertos.

7. Desarrollo del trabajo social comunitario integrado desde la prevención y lograr transformación a través de la capacitación.

\section{REFERENCI AS BI BLI OGRÁFICAS}

1. Partido Comunista de Cuba. Proyecto de Lineamientos de la Política Económica y Social. 1ro. de noviembre de 2010.

2. Yordi García M, Caballero Rivacoba MT. El desarrollo social y el trabajo comunitario. Teoría, metodología y prácticas cubanas. Camagüey: Editorial Ácana; 2009.

3. Dubois A. Capacitación y Formación de Desarrollo de Proyectos Económicos a nivel local en función del Desarrollo Humano Integral. Holguín: Universidad de Holguín; 2006.

4. PNUD. Informe sobre Desarrollo Humano. La Habana: PNUD; 2001.

5. Manfred A. Max-Neef. Desarrollo a Escala Humana. Montevideo: NordanComunidad; 2001. 
6. Castro Ruz R. No tenemos derecho a equivocarnos. Intervención del Presidente Raúl Castro en la Asamblea Nacional del Poder Popular, domingo 20 de diciembre de 2009 [Internet]. LA Habana: Cuba Debate; 2011 [citado 8 abril 2011]. Disponible en: http://www.cubadebate.cu/opinion/2009/12/20/discurso - de-raul-castro-en-laasamblea-nacional/

* Por satisfactores se conciben los factores mediadores mencionados por Max-Neef, como las formas de organización, estructuras políticas, prácticas sociales, condiciones subjetivas, valores, normas, espacios, contextos, comportamientos y actitudes, pero también lo que denomina "bienes", entendidos por él como medios (objetos, artefactos) para potenciar los satisfactores, así como otras condiciones, recursos, medios capaces de favorecer la solución de las carencias y, al mismo tiempo, el aprovechamiento de los potenciales (Las necesidades humanas como contradicciones dialécticas, basado en Max-Neef, 2001).

** Problema, definido por el español J. L. Pinillos como un obstáculo para un propósito o fin, algo que interfiere con una pretendida realización.

$* * *$ Problemas de mediación, no se trata de problemas subjetivos ni materiales sino, principalmente, de funcionamiento o interacción de los elementos de las estructuras o sistemas actuantes en los diversos procesos comunitarios, desde la familia y los comités de defensa de la revolución (CDR) hasta los de servicios sociales como la salud, saneamiento, comercio y gastronomía, transporte, acueducto y otros. Son consecuencia directa de insuficiencias en la integración intersectorial.

Recibido: 28 de octubre de 2010.

Aprobado: 9 de noviembre de 2010.

Rhoden N. Rodríguez Casavielles. Centro Provincial de Higiene, Epidemiología y Microbiología. Calle Libertad No. 36 Reparto Zayas., Holguín, Cuba. Correo electrónico: rhoden@hehg.hlg.sld.cu, obellido@fh.uho.edu.cu 